\title{
Vorwort: Schwerpunkt Musik und Natur
}

Die folgenden beiden Artikel gehen aus der Tagung Music \& Nature hervor, die vom 18.-22. Mai 2011 in Kópavogur, einer Gemeinde bei Reykjavik, abgehalten wurden. Die Tagung war das Ergebnis der deutsch-isländischen Zusammenarbeit zwischen dem Icelandic Music Museum, dem Iceland Music Information Center (IceMic), der Iceland Academy of Music und der Hochschule für Musik und Theater Köln, die von Annette Kreutziger-Herr in Zusammenarbeit mit Katrin Losleben, Florian Heesch, Bjarki Sveinbjörnsson, Sigfridur Björnsdottir und Helgi Jonsson. An der Kooperation waren auch KollegInnen aus Kanada, der Schweiz, Großbritannien, Schweden, Norwegen und Lettland beteiligt.

Initiiert wurde die Tagung im Vorfeld der 17. UN-Klimakonferenz (28.11.10.12.2011), zu der Tausende RegierungsvertreterInnen, NGOs, (Natur-)WissenschaftlerInnen und MedienvertreterInnen in Durban zusammen kommen sollten. Der Klimagipfel stand im Schatten der Kopenhagener Konferenz von 2009 und jener in Cancún 2010, auf denen es nicht gelungen war, eine Nachfolgeregelung für das 2012 auslaufende KyōtoProtokoll zu verabschieden. Diese für viele als dramatisch wahrgenommene Situation löste bei den VeranstalterInnen den Wunsch aus, die Stimme der Kulturwissenschaften und der Künste im Diskursfeld um den Klimawandel zu erheben, was nach den halbgaren Ergebnissen des Klimagipfels in Paris 2015 nicht minder dringlich geworden ist. In der Wissenschaftslandschaft findet dieser Anspruch einen Vorreiter mit den so genannten environmental studies der US-amerikanischen Literaturwissenschaften, wo seit den 1970er Jahren Fragen nach Theorie, AutorIn, Charakteren und Plot von solchen nach der Präsentation und Bedeutung der Umwelt (i. S. von 'place') des Settings abgelöst werden. Ecomusicology ist analog dazu sowohl das künstlerische als auch das (interdisziplinär angelegte) wissenschaftliche Ausloten des Konnexes von Musik bzw. Klang, Kultur und Natur. Hergestellt und analysiert werden Soundscapes, die Verwendung von körperbasierten akustischen Phänomenen (so genannte Biomusik) und die Bedeutung von Umwelt (wieder i. S. von 'place') für die jeweilige Musik. Das Interesse an ökokritischen Fragen kommt aus sämtlichen Sparten des Fachs - von der Ethnomusikologie über die historische Musikwissenschaft hin zu aktiven KünstlerInnen selbst. Spätestens im Anspruch, zur 
Diskussion um den Klimawandel mit Klangkunst beizutragen, den sich verändernden Klang einer schwindenden, weil schmelzender oder anderweitig zurückweichender Natur oder sich mit der Natur verändernde Musiktraditionen zu beschreiben, wird das Thema 'Musik und Natur' politisch. So war es auch von der Ecomusicology Special Interest Group der American Musicological Society gefordert worden: Über die beschreibende und interpretierende Analyse der Verbindung von sound und place hinaus $\mathrm{zu}$ einer politisch-kritischen Herangehensweise zu gelangen. Aber allein die Aufmerksamkeit auf den Themenkomplex 'Natur' (dessen Bedeutungsvielfalt irritieren mag) oder Umwelt in historischer Musik erfüllt die so wichtige Aufgabe, ein ganzheitlicheres Bild von der Musik selbst und vor allem aber der "unendliche[n] Verwicklung" von Natur und Kultur (Seel 2009, 166) zu ermöglichen. Dazu tragen die hier veröffentlichten Aufsätze von Florian Heesch und Maria Birbili bei, die aus Vorträgen der ersten Music and Nature-Tagung im Salurinn Kópavogi hervorgehen. Die in den drei Tagen auf das Thema Natur und Musik aufgefächerten musikhistorischen, ethnomusikologischen und künstlerischen Perspektiven waren so vielfältig, dass unter der Leitung von Katrin Losleben, Florian Heesch, Annette Kreutziger-Herr und Britta Sweers eine Fortsetzung der Tagung unter dem Titel Music and Nature-Reloaded (16.-18.12.2011) in Köln initiiert wurde. Hier wurden die Perspektiven, die erstmals auf Island skizziert worden waren bzw. sich - unter Schlagworten wie Nachhaltigkeit, Gender, Politik und Nationalismus - herauskristallisiert hatten, weiter vertieft.

Ein zentrales Ergebnis der Tagung ist der Band über Music, Climate Change, and the North, der von Britta Sweers herausgegeben und 2017 bei Indiana University Press erscheinen wird. Die hier präsentierten Artikel vermitteln eine Idee von der inhaltlichen Bandbreite des Themas, vor allem aber auch vom politischen Impetus des Themas: Florian Heesch hinterfragt Stereotypien und das vermeintlich dichotome Verhältnis von Kultur und Natur auf Basis von in Schriften und Musik des isländischen Komponisten Jón Leifs herausgearbeiteten vertretenen Ideologien, Maria Birbili benennt die politischen und ökologischen Kontexte polarer und alpiner Opernszenerien in Opern des 19. Jahrhunderts.

Britta Sweers und Katrin Losleben, November 2016 